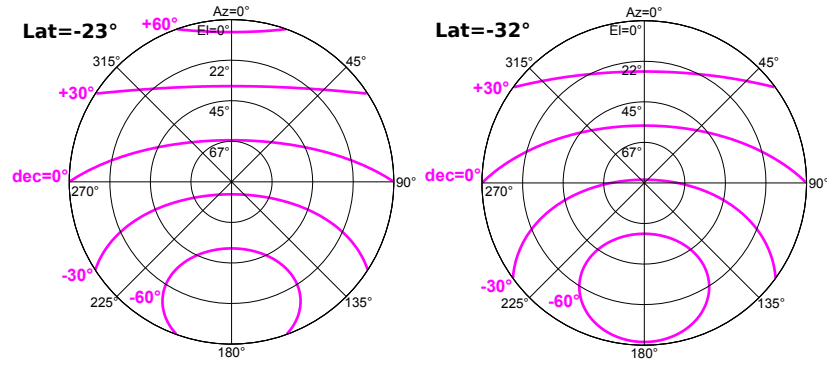

Fig. 6: Loci of constant declination plotted in a polar plot as a function of polar coordinates.

\section{Earth's rotation and polarization diversity}

Our discussion of polarization diversity has mainly focused on the instantaneous polarimetry, that is, the snap-shot mode of the SKA. Obviously, the assumption that the AA does not move means the diurnal rotation of the Earth will itself rotate the element. Thus the SKA will have some polarization diversity for free. However, this does not make the simple polarization diversity scheme discussed in the previous section superfluous. First the diurnal rotation does not apply for snap-shot imaging, which is important for transients. Furthermore the diurnal rotation is with respective to the poles of the celestial sphere, while the boresight of the AAs of the SKA will be towards zenith, so the two types polarization diversity through rotation will be somewhat complementary.

The effect of the diurnal rotation on the polarization diversity of a dual-polarized element can be grasped by considering the trajectory of the fixed sources on celestial sphere in the same sort of polar plots we used previously for the IXR (and orthogonality) of various polarimeters. These are shown in Fig. 4 for two different latitudes, which correspond roughly to the two candidate sites for the SKA. The different magenta lines are for different source declinations. If one overlays these plots over the plots in Fig.s 2, 3, and 5, one sees how the sources at different declinations move through the different beam patterns. One finds that for Vivaldi-type polarimeter patterns, the best alignment is along the cardinal directions, since then one is guaranteed that all visible sources will pass the E- and H-planes of the feeds at the meridian transit. In any case, it is clear that the poles are still sufficiently far away from the boresight, where the polarimetry is best, that the SKA stands to benefit from the polarization diversity scheme suggested here.

\section{Conclusion}

We find that wide-field polarimetry with the AA technology currently considered for the SKA, stands to benefit from polarization diversity. A simple cost-effective scheme for polarization diversity in SKA was proposed and found to improve not only polarimetry but even the overall power received from sources.

Acknowledgements. We wish to thank Stefan Wijnholds for the LOFAR CS10 data and comments on this paper.

\section{References}

W. A. van Cappellen, et al. Low Band Antenna: Architectural Design Document. Technical Report ADD-009, ASTRON, 21 March 2007. 395(3):1558-1568, 2009.

T. D. Carozzi and G. Woan. A generalized measurement equation and van Cittert-Zernike theorem for wide-field radio astronomical interferometry. Monthly Notices of the Royal Astronomical Society,

T. D. Carozzi and G. Woan. A fundamental figure of merit for radio polarimeters. submitted to IEEE trans. Ant. Prop. special issue, 2009.

J. Hamaker. Self-Calibration in the Ska: Dealing with Inherently Strong Instrumental Polarization. In A. B. Smolders \& M. P. van Haarlem, editor, Perspectives on Radio Astronomy: Technologies for Large Antenna Arrays, pages 323-+, 2000.

R. Maaskant, et al. Analysis of Large Microstrip-Fed Tapered Slot Antenna Arrays by Combining Electrodynamic and Quasi-Static Field Models. submitted to IEEE trans. Ant. Prop. special issue.

D. H. Schaubert and T. H. Chio. Wideband Vivaldi Arrays for Large Aperture Antennas. In A. B. Smolders \& M. P. van Haarlem, editor, Perspectives on Radio Astronomy: Technologies for Large Antenna Arrays, pages 49-+, 2000.

R. T. Schilizzi, et al. Preliminary Specifications for the Square Kilometre Array. Technical report SKA memo 100, SKA Program Development Office, 10 December 2007.

S. J. Wijnholds. ITS results 2005: Filling in the gaps. Technical Report RPT-056, ASTRON, 23 September 2005. 


\title{
Effect of atmospheric turbulence in wide-field SKA observations
}

\author{
I. Martí-Vidal ${ }^{1}$, J.C. Guirado ${ }^{2}$, S. Jiménez-Monferrer ${ }^{2}$, and J.M. Marcaide ${ }^{2}$ \\ 1 Max-Planck-Institut für Radioastronomie, Bonn (Germany) \\ 2 Departament d'Astronomia i Astrofísica, Universitat de València (Spain)
}

\begin{abstract}
We present the results of a Monte Carlo study of the astrometric precision and sensitivity of a realization of the SKA in wide-field observations. We consider several effects from the turbulent atmosphere (i.e., ionosphere and wet component of the troposphere) and also from the antenna receivers. We study the changes in dynamic range and astrometric precision as a function of observing frequency and source separation from the image center. We find that, for frequencies between $1 \mathrm{and} 10 \mathrm{GHz}$, it is possible to obtain images with high fidelity, although the atmosphere strongly limits the sensitivity of the instrument compared to the case with no atmosphere. Outside this frequency window, the dynamic range of the images and the accuracy of the source positions decrease. We find that, even if a good a propri model of the atmospheric turbulences (with an accuracy of $\sim 1 \%$ ) is used in the imaging, residual effects from the turbulences can still limit the dynamic ranges of deep, high-contrast $\left(10^{5}-10^{6}\right)$, images.
\end{abstract}

\section{Introduction}

It is well-known that ground-based astronomical observations are affected by the atmosphere. Changes in the atmospheric opacity produce a bias in the source flux density, while dispersive effects distort the shape of the electromagnetic frontwave of the source. Such a distortion translates into a deformation of the observed source structure and/or a variation of the relative positions of all sources observed in a given field. In the case of astronomical devices based on interferometry, dispersive atmospheric effects can be well modelled if the atmosphere above each element of the interferometer (hereafter, station) remains unchanged over the whole portion of the sky being observed. In such cases, the observed visibilities can be calibrated using station-based algorithms, which are relatively simple and computationally inexpensive (e.g. Readhead \& Wilkinson 1978).

However, when the spatial variations of the atmosphere are significant within the observed portion of the sky, as it happens if there are atmospheric turbulences, the opacity and dispersive effects cannot be modeled as a single time-dependent station-based complex gain over the field of view. Unless more complicated calibration algorithms are used (e.g. van der Tol et al. 2007), the effect of these errors on the image are difficult to correct. In this paper, we report on a study of the effects that a turbulent atmosphere may introduce in interferometric observations. We focus our study on the effects produced by turbulences in the dynamic range and astrometric accuracy in wide-field images, as a function of distance to the image center, where the gain calibration of the stations is supposed to be optimized.

We performed simulations of snapshot observations under the effects of a turbulent atmosphere (ionosphere and wet troposphere) and a finite temperature of the antenna receivers. The results here reported are an extension of those earlier reported in Martí-Vidal et al. (2009). In the next section, we describe the details of the array distribution used, as well as the characteristics of the simulated observations. In Sect. 3, we describe how the synthetic noise from the atmosphere and the receivers was added to the visibilities and describe the procedures followed in our Monte Carlo analysis. In Sect. 4, we present the main results obtained; in Sect. 5, we summarize our conclusions.

\section{Details of the simulated observations}

We simulated an interferometric array similar to the planned station distribution of the Square Kilometre Array (SKA). We simulated a total of 200 stations distributed in the following way: $50 \%$ are randomly distributed within a circle of $5 \mathrm{~km} \mathrm{ra}$ dius (inner core); $25 \%$ are distributed outside this circle up to a distance of $150 \mathrm{~km}$ (core), following 5 equiangular spiral arms; the remaining antennae are distributed following the same spiral arms, but up to a distance of $3000 \mathrm{~km}$ from the inner core. This array distribution is similar to that used in $\mathrm{Lal}$ et al. (2009). The curvature of the Earth surface was taken into account in our simulations. We show the resulting array distribution in Fig. 1.

\subsection{Array sensitivity and observing bandwidth}

We simulated interferometric observations using 16 different frequencies, which span in logarithmic bins from $150 \mathrm{MHz}$ to $24 \mathrm{GHz}$ (this is the theoretical frequency window of the SKA). According to Jones (2004), the maximum observing bandwidth of the SKA will be around $25 \%$ of the central observing frequency (up to a maximum bandwidth of $4 \mathrm{GHz}$ for all frequencies above $16 \mathrm{GHz}$ ). This (maximum) frequency-dependent bandwidth translates in our simulations into a changing sensitivity of the SKA as a function of frequency.

The sensitivities of the simulated stations were taken from Jones (2004). These values are set for an elevation of 45 degrees and differ from those given in Schilizzi et al. (2007), but the use of the values given in Schilizzi et al. (2007), instead, does not affect the conclusions of this paper. We interpolated the sensitivities given in Table 1 of Jones (2004) to the fre- 

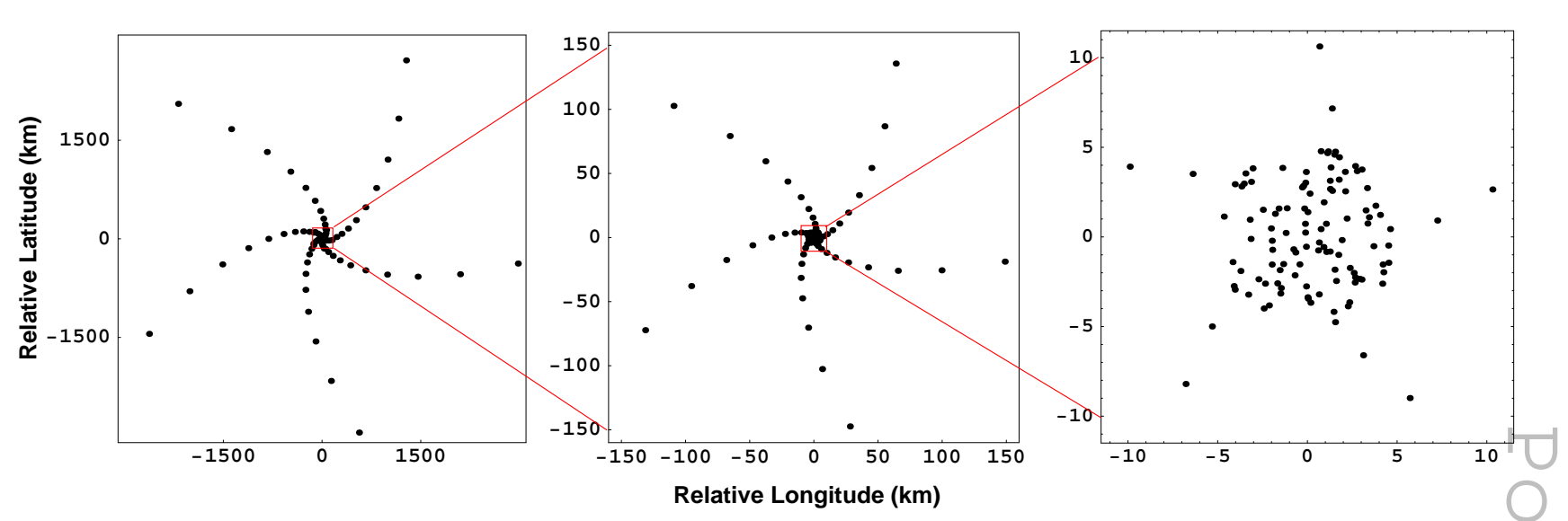

Fig. 1: Array distribution used in our simulations. Axes are relative Longitude (horizontal axis) and Latitude (vertical axis) in km. Left, the $\Omega$ whole array. Center, a zoom to the core. Right, a zoom to the inner core.

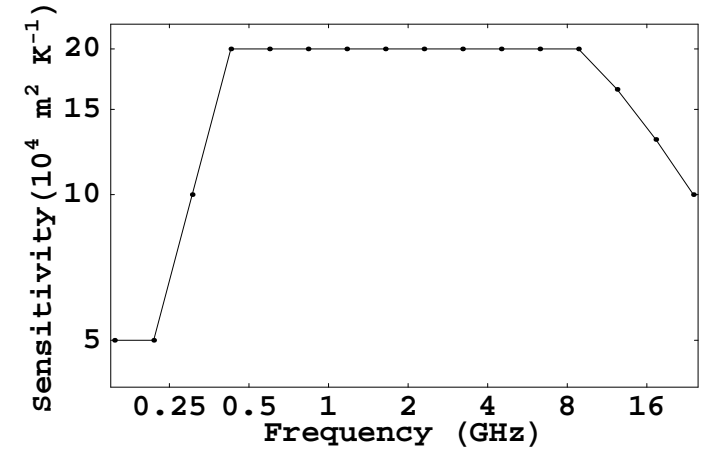

Fig. 2: Station sensitivities (i.e., effective areas over system temperatures) used in our simulations.

quencies used in our simulations. In Fig. 2 we show the station sensitivities used.

\section{Noise model}

The observations were simulated in the following way: we assumed that the phase calibration of the stations is referred to the center of the wide-field image (i.e., the gain calibration of the stations takes into account the atmospheric contribution in the signal from an hypothetic source located at the center of the image); we then determined the image of a source located at a given distance from the image center by computing the differential antenna-gain errors expected at the location of the source.

We implemented two kinds of atmospheric turbulences. The first turbulence was associated to the ionosphere (the free electron content, which introduces dispersion in the radiation) and the other turbulence was associated to the wet troposphere (the water vapour, close to the earth surface, which is in a state of no thermodynamic equilibrium). The effect of ionospheric turbulences on the signal phase varies as $v^{-1}$, affecting the lowfrequency observations; the effect of the wet troposphere on the phase varies as $v$, affecting the high-frequency observations.
The dry troposphere (which is more homogeneously distributed over each station than the wet troposphere) was not considered in our simulations, since the differential effects in the signal phase between the source location and the image center are much smaller than those coming from the water vapor and the ionosphere. Models of the turbulences of the ionosphere and troposphere can be found in many publications (e.g. Thomson et al. 1991). Here, it is suffice to say that these turbulences follow a Kolmogorov distribution. This distribution has a phase structure function given by

$$
D_{\phi}(\theta)=<\left(\Phi\left(\theta_{0}\right)-\Phi\left(\theta_{0}+\theta\right)\right)^{2}>\propto \theta^{5 / 3}
$$

where $\Phi\left(\theta_{0}\right)$ is the phase added by the turbulent screen to the signal of a source located at $\theta_{0}$. The brackets $<\ldots>$ represent averaging over all pointing directions located at a distance $\theta$ from the point located at $\theta_{0}$. The Kolmogorov distribution is fractal-like, so both, ionosphere and wet troposphere, have essentially the same phase distribution, despite of a global scaling factor between them.

The global factors for both distributions (ionosphere and troposphere) were computed according to the typical values of ionospheric and tropospheric conditions. For the ionosphere, the Fried length (i.e., distance in the ionosphere for which the structure function rises to $1 \mathrm{rad}^{2}$ ) was set to $3 \mathrm{~km}$ at $100 \mathrm{MHz}$. For the wet troposphere a Fried length of $3 \mathrm{~km}$ for a frequency of $\sim 22 \mathrm{GHz}$ was used. Since the Kolmogorov distribution is self-similar, it is possible to adapt the results here reported to any other atmospheric conditions (see Sect. 4.2), just accordingly scaling the source separation to the Fried length of the ionosphere (for low-frequency observations) or the wet troposphere (for high-frequency observations). We must notice that the self-similarity of the tropospheric turbulences does not hold for very large scales (the typical baseline lengths in VLBI observations), since there is a saturation in the power spectrum of the distribution (see, e.g., Thomson et al. 1991). However, this is not important in our analysis, since we did not use the absolute phase of the signal coming from a given direction in the sky, but computed the differential effects at each station from two different (closeby) directions, which depend on short- 
scale turbulences. Therefore, the saturation of tropospheric turbulences at large scales does not affect our results.

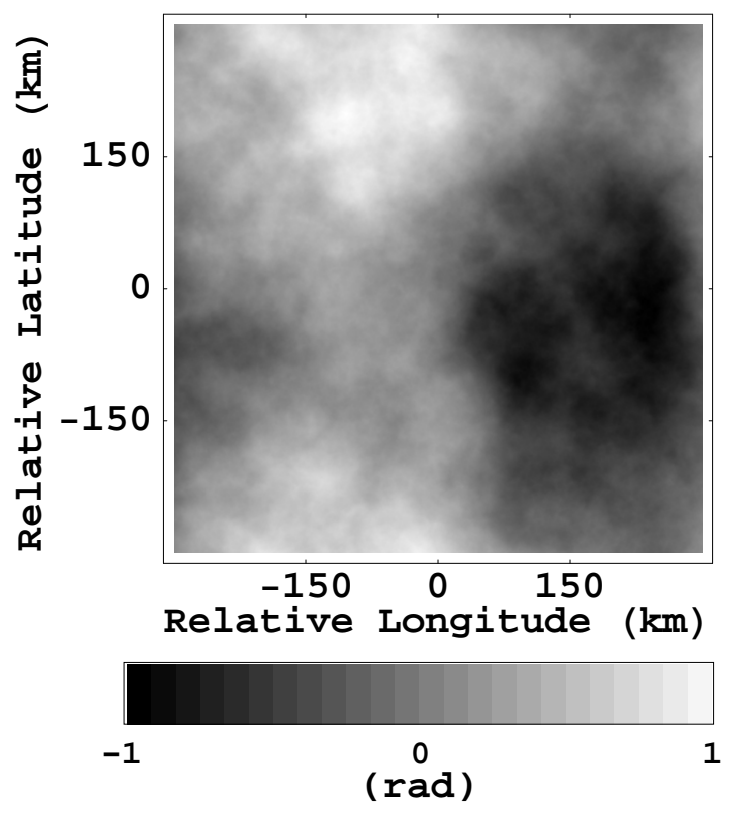

Fig. 3: Example of a turbulent phase screen with Kolmogorov statistics. The grey scale shows variations of optical-path phases, normalized between -1 and 1 radians. The final values of the phases depend (given the self-similarity of the distribution) on a global factor related to the observing frequency and the ionospheric and/or wet tropospheric conditions.

We computed the differential effects from the turbulent atmosphere in two ways. For the antennas of the core (within the central $300 \mathrm{~km}$ ) we generated synthetic phase screens for the ionosphere and troposphere. We show an example of one such screen in Fig. 3. We notice that this figure could represent either ionospheric or tropospheric turbulences in our modelling, just by scaling the screen by the corresponding factor. Two different screens were generated in each Monte Carlo simulation. The screen for simulating the ionosphere was put at a height of $300 \mathrm{~km}$ and the screen for simulating the troposphere was put at a height of $5 \mathrm{~km}$. For the antennas out of the core, we computed the term $\Phi\left(\theta_{0}\right)-\Phi\left(\theta_{0}+\theta\right)$ separately. We proceeded this way (i.e., we generated a phase screen only for the core antennas, thus without generating a much larger screen for the whole array), because the distances between stations out of the core were large enough to ensure that the cross-correlation of turbulences above different stations would be negligible compared to the correlation between those on the calibrator and target source for the same station. This numerical strategy also speeded up our simulations.

It must be noticed that we did not introduce any time evolution of the turbulent phase screens in our simulations. Any evolution of the turbulences could dramatically affect the observations if the acquisition times were larger than the coherence time of the signal, which depends on the evolution of the turbulences and the observing frequency. However, for snapshotlike observations, of the order of a fraction of a minute or so, we could consider, as a good first approximation, a constant turbulence phase screen.

\subsection{Receiver noise model}

Noise from the receivers was added to our model by generating a random gaussian noise in the real and imaginary parts of the visibilities. Such a noise maps into a Rayleigh distribution in the amplitudes and a uniform distribution in the phases if there is no source observed or its flux density is well below the sensitivity of each baseline. The mean deviation, $\sigma$, of the gaussian noise added to the visibilities was (e.g., Thomson et al. 1991, Eq. 6.43):

$$
\sigma=\frac{\sqrt{2} k}{\eta_{Q} \sqrt{\Delta v \Delta t}} \frac{1}{S_{A}}
$$

where $k$ is the Boltzmann constant, $\eta_{Q}$ is the relative loose of signal due to the correlator quantization (we used $\eta_{Q}=0.5$ ), $\Delta v$ is the observing bandwidth, $\Delta t$ is the observing time, and $S_{A}$ is the sensitivity of the stations (collecting area over system temperature, shown in Fig. 2).

\section{Results}

We simulated different sets of observations. In all cases, the observations were snapshots with a duration $t_{0}=60 \mathrm{~s}$. Longer observing times, $t$, would, in principle, increase the dynamic ranges and astrometric precisions shown in all the following sections as $\sqrt{t / t_{0}}$, as long as the changing atmosphere (and, therefore, the changing source positions and shapes) would not introduce important smearing effects in the images after the combination of all the visibilities.

In a first run of simulations, we generated visibilities of targets with flux densities of $0.1,1$, and $10 \mu \mathrm{Jy}$ with a separation of 5 degrees from the image center. A total of 1500 simulations were performed for each flux density and frequency.

In a second run of simulations, we studied the effects of the atmosphere as a function of distance to the image center. For that purpouse, we simulated 1500 observations at $1420 \mathrm{MHz}$ (i.e., the Hydrogen line) of a source with $1 \mu \mathrm{Jy}$ for different separations from the center (2, 3, 4, 5, and 6 degrees).

In a third run of simulations, we used only one Kolmogorov screen (which can represent either ionospheric or tropospheric turbulences, depending on the observing frequency) with different Fried lengths, to study the scalability of the simulations for different image sizes and/or atmospheric conditions.

For each simulated image, obtained by applying uniform weighting to the visibilities, the brightness peak was found and the corresponding point source was subtrated from the visibilities. For the substraction of the point source, the brightness peak was shifted to the nominal position of the source by multiplying the visibilities by the corresponding plane-wave factor in the Fourier plane. Then, the flux density of the point source 
was estimated and the resulting point-source model was substracted from the data. Afterwards, a Fourier inversion of the new visibilities resulted in the image of residuals, from which the root-mean-square (rms) of all the pixels was computed. On the one hand, the deviation of the brightness peak with respect to the nominal position of the source was taken as the astrometry error of that image. On the other hand, the source peak divided by the rms of the residuals was taken as the dynamic range. In Fig. 4 we show the distribution of astrometric deviations and dynamic ranges for the case of a target source of $1 \mu \mathrm{Jy}$ observed at $1420 \mathrm{MHz}$ (which corresponds to an interferometric beam of $\sim 13$ mas) located at 5 degrees from the image center. Once the distributions like those shown in Fig. 4 were obtained, we computed the standard deviation of astrometric corrections and the mean value of dynamic ranges for each source flux density, frequency, and separation. The first quantity was our estimate of the astrometric uncertainty, and the second quantity was an estimate of the achievable dynamic range.
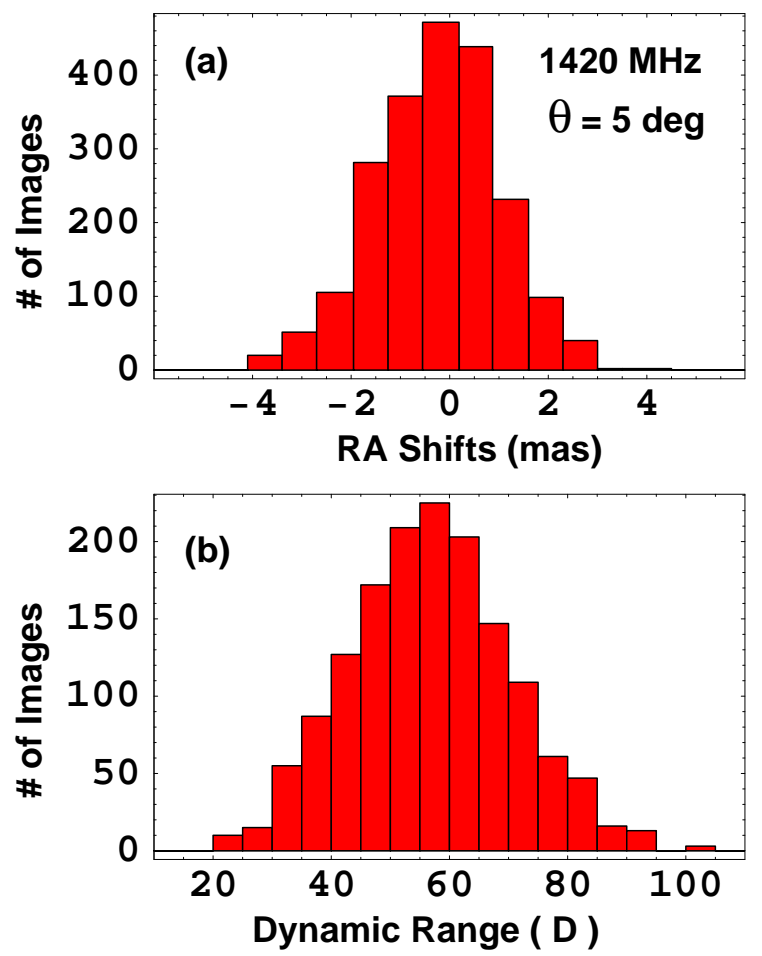

Fig. 4: Distribution of right ascension shifts (a) and dynamic ranges (b) of 1500 snapshot images, simulated at $1420 \mathrm{MHz}$, for a $1 \mu \mathrm{Jy}$ target source located at $5 \mathrm{deg}$ from the image center.

\subsection{Astrometric uncertainty and limited dynamic range}

When turbulent ionosphere and wet troposphere were added to the simulations, we obtained the astrometric uncertainties and dynamic ranges shown in Fig. 5. For very low frequencies (below $\sim 500 \mathrm{MHz}$ ) the ionosphere avoids a clear and pre- cise detection of all sources, no matter their flux densities. For higher frequencies, the astrometric uncertainty decreases notably (mainly because of the dependence of ionospheric effects on the phases as $v^{-1}$ ) and gets limited only by diffraction and sensitivity between 1 and $10 \mathrm{GHz}$ (this frequency window slightly depends on the source flux density, as it can be seen in the figure). For higher frequencies, the wet troposphere begins to affect the astrometric uncertainty, which rises up to around 10 mas for the highest frequencies. We find that the best astrometric accuracy, at least for reasonably well-detected sources, is achieved for frequencies around $4 \mathrm{GHz}$. This is where the ionospheric and (wet) tropospheric components are roughly equal.

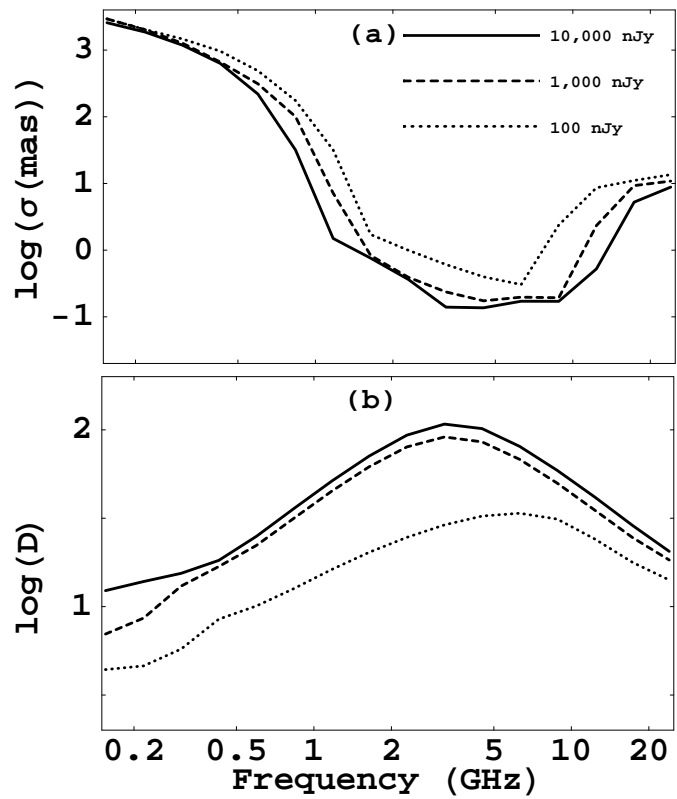

Fig. 5: Astrometric accuracy (a) and dynamic range (b) as a function of frequency, for the case with atmospheric turbulences and a separation of 5 degrees from the image center. Different lines correspond to different target source flux densities $(10 \mu \mathrm{Jy}$, continuous line; $1 \mu \mathrm{Jy}$, dashed line; $0.1 \mu \mathrm{Jy}$, dotted line).

The dynamic range of the images is highly limited by the atmosphere. When the atmosphere adds noise to the visibility phases, there is an extra rms added to the residual images, which depends on the visibility amplitudes, thus limiting the achievable dynamic range no matter the flux density of the source; that is, if the source flux density is higher, the noise of the image will also be higher. This limitation is, of course, more important for the brightest sources. In our case, the brightest source has a flux density of $10 \mu \mathrm{Jy}$. For this source, the maximum dynamic range achieved is only 110, which is $\sim 30$ times smaller than the dynamic range obtained without the atmosphere. This situation can be also understood in another way: the rms of the final image is divided into two components, which are added in quadrature. One component, $\sigma_{t h}$, comes from the receiver noise and is independent of the source 
flux density. The other component, $\sigma_{a t}$, comes from the atmospheric refraction and is equal to a percentage of the source flux density $\left(\sigma_{a t}=K_{r} S\right.$, where $S$ is the source flux density and $K_{r}$ depends on the atmospheric refraction). Hence, the dynamic range, $D$, is

$$
D=\frac{S}{\sqrt{\sigma_{t h}^{2}+\sigma_{a t}^{2}}}=\frac{S}{\sqrt{\sigma_{t h}^{2}+K_{r}^{2} S^{2}}}
$$

For large flux densities $\left(S>>\sigma_{t h}\right)$, the achievable dynamic range will saturate to a value dependent on the atmospheric conditions (i.e., $D \rightarrow 1 / K_{r}$ ) and independent on the source flux density and the sensitivity of the stations. In Fig. 5 we see such a saturation. The dynamic ranges for the $1 \mu \mathrm{Jy}$ and $10 \mu \mathrm{Jy}$ sources are very similar, and the latter should be 10 times higher than the former.

\subsection{Scalability of the simulations and use of a priori turbulence models}

In the previous subsection, we report on the effects of atmospheric turbulences in wide-field interferometric images using fixed values for the Fried lengths of the Kolmogorov distributions of the ionosphere and wet troposphere. Since the Kolmogorov distribution is self-similar, the results reported can be scaled and adapted to other atmospheric conditions. Indeed, these simulations can also be used to estimate the limiting dynamic range and astrometric uncertainty if an a priori model of the tropospheric and/or ionospheric turbulences is used in the imaging. In these cases, the effective Fried length, $r_{e f}$, to compare to our simulations can be estimated as

$$
r_{e f}=r_{0}\left\langle\frac{\phi_{\text {mod }}}{\left|\phi-\phi_{\text {mod }}\right|}\right\rangle
$$

where $r_{0}$ is the real Fried length of the turbulences and the other factor is related to the fractional precision of the a priori turbulence model: $\phi_{\text {mod }}$ is the phase computed from the turbulence model at a given point in the sky and $\phi$ is that corresponding to the real turbulences; the brackets $<\ldots>$ represent averaging over the field of view. If the a priori model of the ionospheric electron distribution is accurate to a given precision level, the effective Fried length of the ionosphere to use will be that corresponding to the residual turbulences (i.e., the difference between the model and the real turbulences). For instance, in the case of a model of the ionospheric electron content with a $99 \%$ accuracy, the effective Fried length for the ionosphere will be $r_{e f}=100 r_{0}$; if the accuracy increases to $99.9 \%, r_{e f}=1000 r_{0}$. In Fig. 6, we show $D_{\text {max }}$, the maximum dynamic range (i.e., for a source with an infinite flux density, so $\sigma_{t h}=0$ in Eq. 1) as a function of $r_{n}$, which we define as the source separation normalized to the Fried length, i.e

$$
r_{n}=\frac{h \sin \theta}{r_{e f}}
$$

In this equation, $h$ is the height of the phase screen and $\theta$ is the separation of the source from the image center. Equation 2 can be used, together with Fig. 6, to compute the maximum achievable dynamic range for many different combinations of source separations, atmospheric conditions, and observing frequencies $\left(r_{e f} \propto v\right.$ for the ionosphere and $r_{e f} \propto v^{-1}$ for the trosposphere). We notice, however, that Fig. 6 has been generated using only one Kolmogorov screen, so it is applicable to either ionospheric dispersion (for low frequencies) or tropospheric dispersion (for high frequencies), but not to a situation where ionospheric and tropospheric effects are similar. In these cases, and as a first approximation, we could set

$$
r_{n}=\frac{h_{\text {ion }} \sin \theta}{\sqrt{r_{\text {ion }}^{2}+\left(\frac{h_{\text {ion }}}{h_{\text {trop }}} r_{\text {trop }}\right)^{2}}}
$$

where $r_{i o n}$ and $r_{\text {trop }}$ are the (effective) Fried lengths of the ionosphere and troposphere, respectively, and $h_{\text {ion }}$ and $h_{\text {trop }}$ are the heights of each phase screen. Also shown in Fig. 6 is the fitting model

$$
D_{\max }=D_{1} r_{n}^{\beta}
$$

where $D_{1}=33.5 \pm 0.2$ and $\beta=-1.016 \pm 0.003$. Figure 6 indicates how difficult is to obtain a high-contrast image with a wide-angle coverage at very low (or high) frequencies. For instance, an image of $10 \times 10$ degrees with a dynamic range of $\sim 10^{5}$ at a frequency of $500 \mathrm{MHz}$ (which translates into $r_{n} \sim$ 1.7 for the ionospheric screen used in the previous subsections) would require, for an observing time of 10 hours (i.e, 600 scans of $60 \mathrm{~s}$ each), a model of the ionospheric turbulence distribution with an accuracy better than $\sim 0.75 \%$ during the whole set of observations.

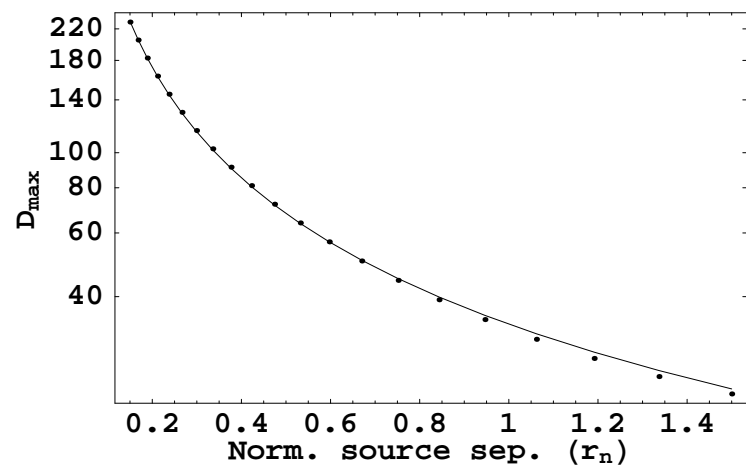

Fig. 6: Maximum dynamic range as a function of source separation normalized to the Fried length of the turbulent phase screen (see Eq. 2) for a 1-scan snapshot $(60 \mathrm{~s})$. The achievable astrometric precision can be estimated as the diffraction limit divided by the dynamic range.

\section{Conclusions}

We report on Monte Carlo estimates of the sensitivity and astrometric precision in wide-field images, obtained with a realization of the SKA, as a function of observing frequency, flux density, and source separation from the image center. Our estimates are based on simulations of snapshot observations, in which we take into account several effects from the turbulent atmosphere and the finite temperature of the receivers. We find 
that the astrometric uncertainty strongly depends on the observing frequency and smoothly increases as the source separation from the image center increases. For frequencies below $\sim 500 \mathrm{GHz}$, ionospheric effects dominate and the astrometry uncertainties (when the source is barely detectable) can be as large as $\sim 1$ as. For frequencies between 1 and $10 \mathrm{GHz}$ (these values slightly depend on the source flux density) atmospheric effects are minimum and we roughly reach the theoretical astrometric precision of the interferometer. Above these frequencies, the wet troposphere begins to dominate and the astrometric uncertainty increases to $\sim 10$ mas for the highest simulated frequency $(25 \mathrm{GHz})$. On the other hand, the dynamic ranges of the images are strongly limited by atmospheric turbulences at all frequencies and for all flux densities (it can decrease, in the worse cases, several orders of magnitude compared to the cases with no atmosphere). Even if good a propri models of the atmospheric turbulences (with accuracies of $\sim 1 \%$ ) are used in the imaging, residual effects from the turbulences can still limit the dynamic ranges of deep, high-contrast $\left(10^{5}-10^{6}\right)$, images.

We propose analytical models for the loose of dynamic range and astrometric accuracy as a function of distance from the image center, and also for the maximum achievable dynamic range as a function of distance to the image center normalized to the Fried length of the turbulent phase screens. This last model can be applied to many different combinations of observing frequency, source separation from the image center, and accuracy of the a priori turbulence model used in the imaging.

Acknowledgements. This work has been supported by the European Community Framework Programme 6, Square Kilometre Array Design Studies (SKADS), contract number 011938. This work has also been partially founded by grants Prometeo 2009/104 of the GVA and AYA2009-13036-CO2-2, AYA2006-14986-CO2-01, and AYA2005-08561-C03 of the Spanish DGICYT. IMV is a fellow of the Alexander von Humboldt Foundation.

\section{References}

Eckers, R.D. 1999, in Synthesis Imaging in Radio Astronomy II (Taylor, Carilli \& Perley, eds.), ASP Conference Series Vol. 180

Jones, D.L. 2004, SKA Memo 45

Lal, D.V., Lobanov, A.P., \& Jiménez-Monferrer, S. 2009, SKA Memo (submitted)

Martí-Vidal, I., Guirado, J.C., Jiménez-Monferrer, S., \& Marcaide, J.M. 2009, SKA Memo 112

Pradel, N., Charlot, P., \& Lestrade, J.-F. 2006, A\&A, 452, 1099

Readhead, A.C.S. \& Wilkinson, P.N. 1978, ApJ, 223, 25

Schilizzi, R.T., Alexander, P., Cordes, J.M., et al. 2007, SKA Memo 100

Thomson, A.R., Moran, J.M., and Swenson, G.W., Interferometry and Synthesis in Radio Astronomy, 1991, Krieger Publ. Corp. (Florida)

van der Tol, S., Jeffs, B.D., \& van der Veen, A.J. 2007, in IEEE

Tr. Signal Processing 